\title{
DiPerna-Lions flow for relativistic particles in an electromagnetic field
}

\author{
P.-E. JABIN ${ }^{1}$ and N. MASMOUDI ${ }^{2}$ \\ ${ }^{1}$ CSCAMM and Dpt of Mathematics, \\ Univ. of Maryland, College Park, MD 20742-4015 \\ email: pjabin@cscamm.umd.edu \\ ${ }^{2}$ Courant Institute \\ 251 Mercer St, New York, NY 10012 \\ email: masmoudi@cims.nyu.edu *
}

\begin{abstract}
We show the existence and uniqueness of a DiPerna-Lions flow for relativistic particles subject to a Lorentz force in an electromagnetic field. The electric and magnetic fields solves the linear Maxwell system in the void but for singular initial conditions. As the corresponding force field is only in $L^{2}$, we have to perform a careful analysis of the cancellations over a trajectory.
\end{abstract}

\section{Introduction}

An important open problem in the theory of renormalized solutions of the Boltzmann equation is to prove global existence for the Boltzmann-Maxwell system. Indeed, even though global renormalized solutions are known to exist for the Boltzmann equation [15] and global weak solutions are known to exist for the Vlasov-Maxwell system [14, there is no such result for the BoltzmannMaxwell system. The main reason is that the two methods of proof turn out

${ }^{*} \mathrm{~N}$. Masmoudi is partially supported by an NSF Grant DMS-0703145 
to be incompatible. Indeed, on the one hand, the existence proof for the Boltzmann equation is based on the theory of renormalized solution and renormalization on the limit equation requires a minimal regularity on the vector fields. More precisely on needs $W^{1, p}$ regularity, $p \geq 1$, see [13], and [2] for the extension to the $B V$ case (we also refer to [11] for a very nice presentation of the main results). On the other hand, the existence proof for the Vlasov-Maxwell system uses a weak compactness argument that only uses averaging lemma and the solution constructed are weak solutions. It seems unclear how to put together the two methods since for the BoltzmannMaxwell system the vector field for the transport equation in the phase space $(x, v)$ is only known to be in $L^{\infty}\left(0, T ; L^{2}\left(\mathbb{R}^{3}\right)\right)$ and hence we can not use the renormalization techniques for the limit.

But of course we are here ignoring the extra structure coming from the fact that the electro-magnetic fields $(E, B)$ solve Maxwell equations with some right-hand side. Let us also remark that for the Boltzmann-Maxwell system with long range interaction (without cut-off), existence of renormalized solutions can be proved since one has only to renormalize the equation on a regularized approximation (before the passage to the limit) and then take advantage of the strong convergence of $f^{n}(t, x, v)$ in all variables to pass to the limit and recover a renormalized solution (see [3]).

The goal of this paper is to give a first step in the understanding of this problem, namely we study the case where $(E, B)$ solve the homogeneous Maxwell system and take advantage of the different speeds of propagation between the slow particles and the fields which propagate at the speed of light.

More precisely, we study the dynamics of relativistic particles in a given electromagnetic field. If one denotes $f(t, x, v)$ the distribution function in phase space, then $f$ solves the kinetic equation

$$
\begin{aligned}
& \partial_{t} f+\frac{v}{\sqrt{1+|v|^{2}}} \cdot \nabla_{x} f+(E+v \times B) \cdot \nabla_{v} f=0, \quad x, v \in \mathbb{R}^{3}, \\
& f(t=0, x, v)=f^{0}(x, v) .
\end{aligned}
$$

for given $E$ and $B$.

Proving well posedness for Eq. (1.1) is a completely open problem if one does not have any regularity for $E$ and $B$, which is the case that we wish to consider here. Therefore we assume that both fields are solution to Maxwell 
equations in the void. As an example, we will just consider the case where

$$
E(t, x)=\partial_{t}\left(t \int_{S^{2}} E_{0}(x+t \omega) d \omega\right), \quad B(t, x)=\partial_{t}\left(t \int_{S^{2}} B_{0}(x+t \omega) d \omega\right) .
$$

Note that if $E_{0}$ and $B_{0}$ are only bounded then $E$ and $B$ have no extra regularity. Nevertheless it is possible to show that

Theorem 1.1 Assume that $E_{0}, B_{0} \in L^{1} \cap L^{2}\left(\mathbb{R}^{3}\right)$ and $f^{0} \in L^{1} \cap L^{\infty}\left(\mathbb{R}^{6}\right)$. Then there exists a unique solution $f \in L^{\infty}\left(\mathbb{R}_{+}, L^{p}\left(\mathbb{R}^{6}\right)\right)$ to Eq. (1.1)

In our context, the first key point is that the particles solve a kinetic equation (instead of a first order general transport equation). It has long been recognized that well posedness for kinetic equations is easier than for general transport equations. The $B V$ case was obtained earlier in [4] (even improved to $B V_{l o c}$ in [19]). While, in the general case, it has been shown that the assumption of $B V$ regularity is optimal (see the counterexample in [12]), it is possible to assume less for equations like (1.1): $H^{3 / 4}$ for instance in [7]. However in more than dimension 1 (i.e. dimension 2 in phase space), $H^{3 / 4}$ is the best that can be done for the moment. Here it is hence necessary to use the additional structure provided on the fields by (1.2).

Of course the result relies on the fact that the particles move with a velocity which is strictly less than the speed of light and hence the wave equation possesses some regularizing properties. This idea was already used in the existence theory of strong solutions to the Vlasov-Maxwell system [21, 6, 17, also this idea is at the origin of the space resonance method used for instance in [16. While this is perfectly satisfactory for the application we have in mind, one could nevertheless wish to study the interaction of particles with fields that are propagated at several different speeds, possibly comparable to the particles' velocity.

We offer a partial answer in an essentially $1-d$ setting in $x$ and now turn to

$$
\begin{aligned}
& \partial_{t} f+\alpha(v) \partial_{x} f+F(t, x) \cdot \nabla_{v} f=0, \quad x \in \mathbb{R}, v \in \mathbb{R}^{d}, \\
& f(t=0, x, v)=f^{0}(x, v) .
\end{aligned}
$$

The function $\alpha$ is assumed to be Lipschitz: $\alpha \in W^{1, \infty}\left(\mathbb{R}^{d}\right)$ and satisfies a genuine non linearity assumption: There exists $C$ such that for all $w \in \mathbb{R}$ and $\eta>0$, we have

$$
|\{v,|\alpha(v)-w| \leq \eta\}| \leq C \eta .
$$


The force field $F$ is assumed to be given by

$$
F(t, x)=\sum_{n} F^{0}\left(x-\xi_{n} t\right) \mu_{n}
$$

with $F^{0} \in L^{\infty}$, together with the bound on the $\mu_{n}$

$$
\exists \gamma>2, \quad \sum_{n}\left(1+n^{\gamma}\right) \mu_{n}<\infty
$$

Note that whereas $x$ is necessarily 1 dimensional, there is no such constraint on $v$. So for instance, Eq. (1.1) in the radially symmetric case would fit in this reduced framework.

We have

Theorem 1.2 Assume (1.6), then for any $f^{0} \in L^{p}\left(\mathbb{R} \times \mathbb{R}^{d}\right)$ for $p>1$, there exists a unique solution $f \in L^{\infty}\left(\mathbb{R}_{+}, L^{p}\left(\mathbb{R} \times \mathbb{R}^{d}\right)\right.$ to Eq. (1.3) where $F$ is given by (1.5).

In this 1 dimensional context, many results are already known. If $v \in \mathbb{R}$, $\alpha(v)=v$ and $F(t, x)=F^{0}(x)$ is autonomous then well posedness was already obtained in [5], with an extension when $F^{0}$ is only $L^{p}$ in [18]. The key for both results is the Hamiltonian structure which implies the propagation of the total energy $v^{2} / 2+\Phi(x)$ with $-\Phi^{\prime}=F^{0}$ which allows to compute $v$ in terms of $x$ (up to a sign).

This type of result was extended to general, autonomous transport equations in dimension 2 with a force field of bounded divergence, which is hence "close enough" in some sense to the Hamiltonian case. An additional assumption of noncharacteristic curve is also needed (this would correspond to (1.4) here); we refer to [9], 8] and [1] which has the most general assumptions.

Eq. (1.3) is still a kinetic equation: Even though strictly speaking, we are not in a Hamiltonian case as $v \in \mathbb{R}^{d}$, it is very close to the earlier formulation of [5] or [18] (more than the later extensions). But Theorem 1.2] is not limited to autonomous $F$ which is the real improvement here. Unfortunately it is still not as general as one would like as we still have to assume some structure on the time dependence of $F$ given by (1.5)-(1.6) . 


\section{Proof of Theorem 1.1}

\subsection{Definition of the functional and reduction of the problem}

As the structure of $E$ and $B$ is essentially the same, we only study the following equation

$$
\partial_{t} f+\frac{v}{\sqrt{1+v^{2}}} \cdot \nabla_{x} f+F(t, x, v) \cdot \nabla_{v} f=0,
$$

with

$$
F(t, x, v)=\nu(v) \partial_{t}\left(t \int_{S^{2}} F_{0}(x+t \omega) d \omega\right),
$$

with $\nu$ a $C^{\infty}$ function of $v$.

Following the now classical connections between transport equations and ordinary differential equations, described in [13] or [2], Theorem 1.1]is implied by the existence and uniqueness of flows to the ODE

$$
\begin{aligned}
& \frac{d}{d t} X(t, x, v)=\frac{V(t, x, v)}{\sqrt{1+V^{2}(t, x, v)}}, \quad \frac{d}{d t} V(t, x, v)=F(t, X), \\
& X(0, x, v)=x, \quad V(0, x, v)=v .
\end{aligned}
$$

As flows the solutions are required to satisfy

Property 1 For any $t \in \mathbb{R}$ the application

$$
(x, v) \in \mathbb{R}^{3} \times \mathbb{R}^{3} \mapsto(X(t, x, v), V(t, x, v)) \in \mathbb{R}^{3} \times \mathbb{R}^{3}
$$

is globally invertible and has Jacobian 1 at almost every $(x, v) \in \mathbb{R}^{3} \times \mathbb{R}^{3}$. It also defines a semi-group

$$
\begin{array}{ll}
\forall s, t \in \mathbb{R}, & X(t+s, x, v)=X(s, X(t, x, v), V(t, x, v)), \\
\text { and } & V(t+s, x, v)=V(s, X(t, x, v), V(t, x, v)) .
\end{array}
$$

Theorem 1.1 is then equivalent to

Theorem 2.1 Assume that $F$ satisfies Equation (2.2) with initial data $F_{0} \in$ $L^{1} \cap L^{2}\left(\mathbb{R}^{3}\right)$. Then there exists a unique solution to (2.3) satisfying Property 1 . 
Our strategy here is to derive explicit quantitative estimates on the trajectory. A functional was introduced in [10] for that (see also [20] for an extension). We use here the modified functional introduced in [7] specifically for kinetic equations: For any compact domain $\Omega \subset \mathbb{R}^{6}$ we look at

$$
\begin{aligned}
Q_{\delta}(T)=\int_{\Omega} \log \left(1+\frac{1}{|\delta|^{2}}\right. & {\left[\left(\sup _{0 \leq t \leq T}\left|X(t, x, v)-X^{\delta}(t, x, v)\right|^{2}\right.\right.} \\
+ & \left.\left.\left.\int_{0}^{T}\left|V(t, x, v)-V^{\delta}(t, x, v)\right|^{2} d t\right) \wedge 1\right]\right) d x d v
\end{aligned}
$$

where $(X, V)$ is a solution to (2.3) (or a regularized version) satisfying Property 1 and $\left(X^{\delta}, V^{\delta}\right)$ is either a solution to a regularized version of (2.3) or verifies

$$
\begin{aligned}
\exists\left(\delta_{1}, \delta_{2}\right) \in \mathbb{R}^{6} \quad \text { with }\left|\left(\delta_{1}, \delta_{2}\right)\right| \leq \delta, \\
\left(X^{\delta}, V^{\delta}\right)(t, x, v)=(X, V)\left(t, x+\delta_{1}, v+\delta_{2}\right) .
\end{aligned}
$$

In the following, we will frequently abuse the notation $\delta$ for in fact $|\delta|$. Then Theorem 1.1 is implied by

Proposition 2.1 For any $\Omega$ compact, any $F_{0} \in L^{1} \cap L^{2}\left(\mathbb{R}^{3}\right)$, there exists a function $\psi$ depending on $T, \Omega$ and $F^{0}$, such that for any $(X, V)$ solution to (2.3) with $F$ given by Equation (1.2), satisfying Property 1 , and $\left(X^{\delta}, V^{\delta}\right)$ satisfying (2.6),

$$
Q_{\delta}(T) \leq T \psi(-\log |\delta|)
$$

with

$$
\frac{\psi(\xi)}{\xi} \longrightarrow 0, \quad \text { as } \xi \rightarrow \infty .
$$

The connection between Proposition 2.1 and Theorem 2.1 is simple and we refer to [10] or [7] for a detailed explanation. Note however that it is not possible to obtain a direct Lipschitz estimate here and it is necessary to distinguish between $X-X^{\delta}$ and $V-V^{\delta}$ as in $Q_{\delta}$.

\subsection{Proof of Proposition 2.1: First steps}

\subsubsection{Truncation of large velocities}

First note that, by the usual estimates on solutions to wave equations, since $F_{0}$ is in $L^{1} \cap L^{2}$ then so is $F(t, x)$ and $B$. Indeed

$$
F(t, x)=\int_{S^{2}} F_{0}(x+t \omega) d \omega+t \int_{S^{2}} \omega \cdot \nabla_{x} F_{0}(x+t \omega) d \omega .
$$


The bound is obviously true for the first term. As for the second, applying Fourier transform in $x$ yields

$$
\mathcal{F} t \int_{S^{2}} \omega \cdot \nabla_{x} F_{0}(.+t \omega) d \omega=\hat{F}_{0}(\xi) t \int_{S^{2}} e^{i t \xi \cdot \omega} \omega \cdot \xi d \omega=\hat{F}_{0}(\xi) M_{t}(\xi) .
$$

The multiplier $M_{t}(\xi)$ is bounded uniformly in $t$.

This means that for any $K$,

$$
\begin{aligned}
\int_{\Omega} \mathbb{I}_{\left\{(x, v)\left|\int_{0}^{T}\right| F(t, X(t, x, v)) \mid d t \geq K\right\}} d x d v \\
\quad \leq \frac{1}{K^{2}} \int_{\Omega} \int_{0}^{T}|F(t, X(t, x, v))|^{2} d t d x d v \leq C \frac{T}{K^{2}}
\end{aligned}
$$

Therefore denoting by $\Omega_{K}$

$$
\begin{aligned}
& \Omega_{K}=\{(x, v) \in \Omega \text { s.t. } \quad \int_{0}^{T}|F(t, X(t, x, v))| d t \leq K \\
&\text { and } \left.\quad \int_{0}^{T}\left|F\left(t, X^{\delta}(t, x, v)\right)\right| d t \leq K\right\},
\end{aligned}
$$

one deduces that $\left|\Omega \backslash \Omega_{K}\right| \leq C T / K^{2}$ and hence

$$
Q_{\delta}(T) \leq \frac{C T}{K^{2}}|\log \delta|+Q_{\delta}^{K}(T)
$$

with

$$
\begin{aligned}
Q_{\delta}^{K}(T)=\int_{\Omega_{K}} \log \left(1+\frac{1}{|\delta|^{2}}(\right. & \sup _{0 \leq t \leq T}\left|X(t, x, v)-X^{\delta}(t, x, v)\right|^{2} \\
& \left.\left.+\int_{0}^{T}\left|V(t, x, v)-V^{\delta}(t, x, v)\right|^{2} d t\right)\right) d x d v
\end{aligned}
$$

Finally note that for $(x, v)$ in $\Omega_{K}$

$$
|V(t, x, v)| \leq|v|+K, \quad|X(t, x, v)| \leq|x|+t|v|+t K .
$$

As $(x, v) \in \Omega$ which is compact then for some constant $C,|V|+|X| \leq C K$ and the same is of course true for $X^{\delta}$ and $V^{\delta}$. 


\subsubsection{The free transport contribution}

Now let

$$
\begin{aligned}
A_{\delta}(t, x, v)= & |\delta|^{2}+\sup _{0 \leq s \leq t}\left|X(s, x, v)-X^{\delta}(s, x, v)\right|^{2} \\
& +\int_{0}^{t}\left|V(s, x, v)-V^{\delta}(s, x, v)\right|^{2} d s .
\end{aligned}
$$

Compute

$$
\begin{aligned}
& \frac{d}{d t} \log \left(1+\frac{1}{|\delta|^{2}}\left(\sup _{0 \leq s \leq t}\left|X(s, x, v)-X^{\delta}(s, x, v)\right|^{2}\right.\right. \\
& \left.\left.\quad+\int_{0}^{t}\left|V(s, x, v)-V^{\delta}(s, x, v)\right|^{2} d s\right)\right) \\
& =\frac{2}{A_{\delta}(t, x, v)}\left(\frac{d}{d t}\left(\sup _{0 \leq s \leq t}\left|X(s, x, v)-X^{\delta}(s, x, v)\right|^{2}\right)+\left(V(t)-V^{\delta}(t)\right)\right. \\
& \left.\quad \times \int_{0}^{t}\left(F(s,(X, V)(s, x, v))-F\left(s,\left(X^{\delta}, V^{\delta}\right)(s, x, v)\right)\right) d s\right) .
\end{aligned}
$$

Recall that for any $f \in B V(0, T)$, we have

$$
\frac{d}{d t}\left(\max _{0 \leq s \leq t} f(s)^{2}\right) \leq 2\left|f(t) f^{\prime}(t)\right| \leq 4|f(t)|^{2}+\frac{1}{2}\left|f^{\prime}(t)\right|^{2} .
$$

And in addition

$$
\left|\partial_{t} X-\partial_{t} X^{\delta}\right|^{2}=\left|V / \sqrt{1+|V|^{2}}-V^{\delta} / \sqrt{1+\left|V^{\delta}\right|}\right|^{2} \leq 4\left|V-V^{\delta}\right|^{2} .
$$

Hence we deduce from the previous computation that

$$
\begin{aligned}
Q_{\delta}^{K}(T) & \leq \int_{\Omega_{K}} \int_{0}^{T} \frac{8\left|X-X^{\delta}\right|^{2}+\left|V-V^{\delta}\right|^{2} / 2}{A_{\delta}(t, x, v)} d t d x d v+\tilde{Q}_{\delta}(T) \\
& \leq 8|\Omega|(1+T)+\tilde{Q}_{\delta}(T)+\frac{1}{2} \int_{\Omega_{K}} \int_{0}^{T} \frac{\left|V-V^{\delta}\right|^{2}}{A_{\delta}(t, x, v)} d t d x d v
\end{aligned}
$$

where,

$$
\begin{aligned}
\tilde{Q}_{\delta}(T)=2 & \int_{\Omega_{K}} \int_{0}^{T} \frac{V^{\delta}(t, x, v)-V(t, x, v)}{A_{\delta}(t, x, v)} . \\
& \int_{0}^{t}\left(F\left(s,\left(X^{\delta}, V^{\delta}\right)(s, x, v)-F(s,(X, V)(s, x, v))\right) d s d t d x d v .\right.
\end{aligned}
$$


Remark that

$$
\begin{aligned}
\int_{\Omega_{K}} \int_{0}^{T} \frac{\left|V-V^{\delta}\right|^{2}}{A_{\delta}(t, x, v)} d t d x d v & \leq \int_{\Omega_{K}} \int_{0}^{T} \frac{\partial_{t} A_{\delta}(t, x, v)}{A_{\delta}(t, x, v)} d t d x d v \\
& \leq \int_{\Omega_{K}} \log \left(\frac{A_{\delta}(T, x, v)}{|\delta|^{2}}\right) d x d v \\
& \leq Q_{\delta}^{K}(T),
\end{aligned}
$$

where we recall that $A_{\delta}(0, x, v)=\delta^{2}$. Therefore, we have

$$
Q_{\delta}^{K}(T) \leq 8|\Omega|(1+T)+2 \tilde{Q}_{\delta}(T)
$$

and it is enough to bound $\tilde{Q}_{\delta}(T)$.

For technical reasons related to some interpolations that will be explained later, we will bound a more general term than $\tilde{Q}_{\delta}$, namely

$$
\begin{aligned}
\bar{Q}_{\delta}(T)= & 2 \int_{\Omega_{K}} \int_{0}^{T} \frac{V \delta(t, x, v)-V(t, x, v)}{A_{\delta}(t, x, v)} . \\
& \int_{0}^{t}\left(\nu\left(V_{s}^{\delta}\right) G\left(s, X^{\delta}(s, x, v)\right)-\nu\left(V_{s}\right) G(s, X(s, x, v))\right) d s d t d x d v
\end{aligned}
$$

where we assume that $G$ satisfies the same assumption as $F$, namely

$$
G(t, x)=\partial_{t}\left(t \int_{S^{2}} G^{0}(x-\omega t) d \omega\right) .
$$

In the term $\bar{Q}_{\delta}$ we decouple the connection between the dynamics of $(X, V)$ and $\left(X^{\delta}, V^{\delta}\right)$ which is related to $F$ and the $G$ function which appears in $\bar{Q}_{\delta}$. This means that $\bar{Q}_{\delta}$ is now linear in $G^{0}$ which will later allow us to use interpolation theory.

Let us remark that

$$
\begin{aligned}
\bar{Q}_{\delta}(T) \leq & \int_{\Omega_{K}} \int_{0}^{T}\left(\nu(V)+\nu\left(V^{\delta}\right)\right) \frac{V(t, x, v)-V^{\delta}(t, x, v)}{A_{\delta}(t, x, v)} \\
& \int_{0}^{t}\left(G\left(s, X_{s}^{\delta}\right)-G\left(s, X_{s}\right)\right) d s d x d v d t \\
+ & \int_{\Omega_{K}} \int_{0}^{T}\left(\nu\left(V_{t}\right)-\nu\left(V_{t}^{\delta}\right)\right) \frac{V(t, x, v)-V^{\delta}(t, x, v)}{A_{\delta}(t, x, v)} \\
& \int_{0}^{t}\left(G\left(s, X_{s}^{\delta}\right)+G\left(s, X_{s}\right)\right) d s d x d v d t
\end{aligned}
$$


As $\nu$ is lipschitz the second term may be directly bounded by

$$
\begin{aligned}
C_{K} \int_{\Omega_{K}} \int_{0}^{T} \frac{\left|V(t, x, v)-V^{\delta}(t, x, v)\right|^{2}}{A_{\delta}(t, x, v)} & \\
& \int_{0}^{t}\left(\left|G\left(s, X_{s}^{\delta}\right)\right|+\left|G\left(s, X_{s}\right)\right|\right) d s d x d v d t,
\end{aligned}
$$

where $C_{K}=\max _{B(0, C K)}|\nabla \nu(K)|$. Note that

$$
\int_{s}^{T} \frac{\left|V(t, x, v)-V^{\delta}(t, x, v)\right|^{2}}{A_{\delta}(t, x, v)} d t \leq \int_{s}^{T} \frac{\partial_{t} A_{\delta}}{A_{\delta}} d t \leq-C \log |\delta| .
$$

Hence by Fubini

$$
\bar{Q}_{\delta}(T) \leq 2 I_{\delta}(T)+C_{K} T|\log | \delta||\left(\left\|G^{0}\right\|_{L^{1}}+\left\|G^{0}\right\|_{L^{2}}\right),
$$

with

$$
\begin{aligned}
I_{\delta}=\nu(K) & \int_{\Omega_{K}} \int_{0}^{T} \frac{\left|V(t, x, v)-V^{\delta}(t, x, v)\right|}{A_{\delta}(t, x, v)} \\
& \left|\int_{0}^{t}\left(G\left(s, X_{s}^{\delta}\right)-G\left(s, X_{s}\right)\right) d s\right| d x d v d t .
\end{aligned}
$$

\subsection{Proof of Proposition 2.1: The main bound}

The term $I_{\delta}$ is quite technical to bound and we hence summarize the computations in the following lemma

Lemma 2.1 For any $G$ satisfying (2.9) with $G_{0} \in L^{1} \cap L^{2}\left(\mathbb{R}^{3}\right)$ and any $(X, V)$ solution to (2.3) with (11), any $\left(X^{\delta}, V^{\delta}\right)$ verifying (2.6), there exists a constant $C$ depending only on $T$, s.t.

$$
I_{\delta} \leq C \nu(K) K^{10}|\log | \delta \|\left(\left\|G^{0}\right\|_{L^{1}}+\left\|G^{0}\right\|_{L^{2}}\right)\left(1+\left\|F^{0}\right\|_{L^{2}}\right) .
$$

\section{Beginning of the proof of Lemma 2.1. Write}

$$
\begin{aligned}
\int_{0}^{t}\left(G\left(s, X_{s}^{\delta}\right)-\right. & \left.G\left(s, X_{s}\right)\right) d s=\int_{0}^{t} \int_{S^{2}}\left(G^{0}\left(X_{s}-\omega s\right)-G^{0}\left(X_{s}^{\delta}-\omega s\right)\right) d \omega d s \\
& +\int_{0}^{t} \int_{S^{2}}\left(\omega \cdot \nabla_{x} G^{0}\left(X_{s}-\omega s\right)-\omega \cdot \nabla_{x} G^{0}\left(X_{s}^{\delta}-\omega s\right)\right) s d \omega d s .
\end{aligned}
$$


Introduce the two changes of variables

$$
\Phi_{X}(s, \omega)=X_{s}-s \omega, \quad \Phi_{X^{\delta}}(s, \omega)=X_{s}^{\delta}-s \omega .
$$

The Jacobian of the transform is

$$
J_{X}=C s^{2}\left|\dot{X}_{s} \cdot \omega-1\right|,
$$

and the corresponding formula for $J_{X^{\delta}}$. Denote $\left(s_{X}, \omega_{X}\right)(z)$ the inverse of $\Phi_{X}$, namely $z=X_{s_{X}(z)}-s_{X}(z) \omega_{X}(z)$ and $O_{X}^{t}=\bigcup_{s \in[0, t], \omega \in S^{2}} \Phi_{X}(s, \omega)$. One can easily prove that

$$
O_{X}^{t}=B(X(t, x, v), t) .
$$

One inclusion is indeed obvious and as for the other one, note that

$$
\left|\Phi_{X}(s, \omega)-X(t)\right| \leq s+|X(s)-X(t)| \leq s+|t-s|
$$

as $|\dot{X}|<1$.

One obtains

$$
\left|\int_{0}^{t}\left(G\left(s, X_{s}^{\delta}\right)-G\left(s, X_{s}\right)\right) d s\right| \leq A+|B|+|C|+D+|E|
$$

with

$$
\begin{aligned}
A= & \int_{O_{X}^{t} \backslash O_{X^{\delta}}^{t}}\left|G^{0}(z)\right| \frac{C d z}{s_{X}^{2}\left|\dot{X}_{s} \cdot \omega_{X}-1\right|} \\
& +\int_{O_{X^{\delta}}^{t} \backslash O_{X}^{t}}\left|G^{0}(z)\right| \frac{C d z}{s_{X^{\delta}}^{2}\left|\dot{X}_{s}^{\delta} \cdot \omega_{X^{\delta}}-1\right|} \\
B= & \int_{O_{X}^{t} \cap O_{X^{\delta}}^{t}} G^{0}(z)\left(\frac{C}{s_{X}^{2}\left|\dot{X}_{s_{X}} \cdot \omega_{X}-1\right|}-\frac{C}{s_{X^{\delta}}^{2}\left|\dot{X}_{s_{X^{\delta}}}^{\delta} \cdot \omega_{X^{\delta}}-1\right|}\right) d z
\end{aligned}
$$

which correspond to the term without derivative,

$$
\begin{aligned}
C= & \int_{\partial B(X(t), t)} G^{0}(z) \frac{C}{s_{X}\left|\dot{X}_{s_{X}} \cdot \omega_{X}-1\right|} d S(z) \\
& -\int_{\partial B\left(X^{\delta}(t), t\right)} G^{0}(z) \frac{C}{s_{X^{\delta}}\left|\dot{X}_{s_{X^{\delta}}^{\delta}} \cdot \omega_{X^{\delta}}-1\right|} d S(z) \\
D= & \int_{O_{X}^{t} \backslash O_{X^{\delta}}^{t}}\left|G^{0}(z)\right|\left|\nabla_{z} \cdot\left(\omega_{X} \frac{C}{s_{X}\left|\dot{X}_{s} \cdot \omega_{X}-1\right|}\right)\right| d z \\
& +\int_{O_{X^{\delta}}^{t} \backslash O_{X}^{t}}\left|G^{0}(z)\right|\left|\nabla_{z} \cdot\left(\omega_{X^{\delta}} \frac{C}{s_{X^{\delta}}^{2}\left|\dot{X}_{s}^{\delta} \cdot \omega_{X^{\delta}}-1\right|}\right)\right| d z,
\end{aligned}
$$


and finally

$$
\begin{aligned}
E=\int_{O_{X}^{t} \cap O_{X^{\delta}}^{t}} G^{0}(z) & \left(\nabla_{z} \cdot\left(\frac{C \omega_{X}}{s_{X}\left|\dot{X}_{s_{X}} \cdot \omega_{X}-1\right|}\right)\right. \\
& \left.-\nabla_{z} \cdot\left(\frac{C \omega_{X^{\delta}}}{s_{X^{\delta}}\left|\dot{X}_{s_{X^{\delta}}^{\delta}} \cdot \omega_{X^{\delta}}-1\right|}\right)\right) d z .
\end{aligned}
$$

Note that $C$ is a boundary term coming from the integration by parts. We hope there is no confusion since $C$ is used for constants that may change from one line to the other. We denote by $I_{A}, \ldots, I_{E}$ the integrals, over $\Omega_{K} \times[0, T]$ of the previous quantities multiplied by

$$
\left(\nu\left(V_{t}\right)+\nu\left(V_{t}^{\delta}\right)\right) \frac{\left|V(t, x, v)-V^{\delta}(t, x, v)\right|}{A_{\delta}(t, x, v)} .
$$

\subsubsection{Bound for $I_{B}$}

As part of the bound for $I_{E}$ uses the same steps, we prove here a more general result on quantities like $I_{B}$. We call them $I_{B m o d}$.

Lemma 2.2 Assume that $H \in W^{1, \infty}$ and that $4 / 3<p<2$, then one has for some constant $C$ depending on $p$ and the norm of $H$ and for $k=1,2$,

$$
\begin{aligned}
& I_{B m o d}:= \nu(K) \int_{0}^{T} \int_{\Omega_{K}} \frac{\left|V_{t}-V_{t}^{\delta}\right|}{A_{\delta}(t, x, v)} \int_{O_{X}^{t} \cap O_{X^{\delta}}^{t}}\left|G^{0}(z)\right| \mid \frac{H\left(s_{X}, \omega_{X}\right)}{s_{X}^{2}\left|\dot{X}_{s_{X}}^{\delta} \cdot \omega_{X}-1\right|^{k}} \\
&-\frac{H\left(s_{X^{\delta}}, \omega_{X^{\delta}}\right)}{s_{X^{\delta}}^{2}\left|\dot{X}_{s_{X^{\delta}}} \cdot \omega_{X^{\delta}}-1\right|^{k}} \mid d z d x d v d t \\
& \leq C K^{5+k} \nu_{K} \sqrt{-\log |\delta|}\left(\left\|G^{0}\right\|_{L^{1}}+\left\|G^{0}\right\|_{L^{p}}\right) .
\end{aligned}
$$

Lemma 2.2 with $k=1$ and $H$ constant implies that for $4 / 3<p \leq 2$, we have

$$
I_{B} \leq C \nu(K) K^{6} \sqrt{-\log |\delta|}\left(\left\|G^{0}\right\|_{L^{1}}+\left\|G^{0}\right\|_{L^{p}}\right) .
$$

Proof of Lemma 2.2. Denote

$$
B m o d=\int_{O_{X}^{t} \cap O_{X^{\delta}}^{t}}\left|G^{0}(z)\right|\left|\frac{H\left(s_{X}, \omega_{X}\right)}{s_{X}^{2}\left|\dot{X}_{s_{X}} \cdot \omega_{X}-1\right|^{k}}-\frac{H\left(s_{X^{\delta}}, \omega_{X^{\delta}}\right)}{s_{X^{\delta}}^{2}\left|\dot{X}_{s_{X^{\delta}}}^{\delta} \cdot \omega_{X^{\delta}}-1\right|^{k}}\right| d z .
$$


Recall that $\dot{X}_{s}=V_{s} / \sqrt{1+V_{s}^{2}}$ and that $\left|V_{s}\right| \leq C K$, so that $\left|\dot{X}_{s}\right| \leq / 1-$ $1 /(C K)$ and $\left|\dot{X}_{s} \cdot \omega-1\right| \geq 1 /(C K)$. Then

$$
\begin{array}{r}
\left|\frac{H\left(s_{X}, \omega_{X}\right)}{s_{X}^{2}\left|\dot{X}_{s} \cdot \omega-1\right|^{k}}-\frac{H\left(s_{X^{\delta}}, \omega_{X^{\delta}}\right)}{s_{X^{\delta}}^{2}\left|\dot{X}_{s}^{\delta} \cdot \omega_{X^{\delta}}-1\right|^{k}}\right| \leq C K^{k}\left(\left|s_{X}-s_{X^{\delta}}\right|\left(\frac{1}{s_{X}^{3}}+\frac{1}{s_{X^{\delta}}^{3}}\right)\right. \\
\left.\quad+K\left|\omega_{X}-\omega_{X^{\delta}}\right|\left(\frac{1}{s_{X}^{2}}+\frac{1}{s_{X^{\delta}}^{2}}\right)+K \frac{\left|V_{s_{X}}-V_{s_{X}}^{\delta}\right|}{s_{X}^{2}}+K \frac{\mid V_{s_{X} \delta}-V_{s_{X} \delta}^{\delta}}{s_{X^{\delta}}^{2}}\right) .
\end{array}
$$

On the other hand, by definition $X_{s_{X}}-s_{X} \omega_{X}=X_{s_{X^{\delta}}}^{\delta}-s_{X^{\delta}} \omega_{X^{\delta}}$, so

$\Phi_{X}\left(s_{X}, \omega_{X}\right)-\Phi_{X}\left(s_{X^{\delta}}, \omega_{X^{\delta}}\right)=X_{s_{X}}-X_{s_{X^{\delta}}}-s_{X} \omega_{X}+s_{X^{\delta}} \omega_{X^{\delta}}=X_{s_{X^{\delta}}}^{\delta}-X_{s_{X^{\delta}}}$.

Recall that $\left|\omega_{X}\right|=\left|\omega_{X^{\delta}}\right|=1$. Therefore $\left|s_{X} \omega_{X}-s_{X^{\delta}} \omega_{X^{\delta}}\right| \geq\left|s_{X}-s_{X^{\delta}}\right|$ and as $\left|X_{s_{X}}-X_{s_{X} \delta}\right| \leq(1-1 /(C K))\left|s_{X}-s_{X^{\delta}}\right|$,

$$
\left|s_{X}-s_{X^{\delta}}\right| \leq \max _{s \leq \inf \left(s_{X}, s_{X^{\delta}}\right)}\left|X_{s}-X_{s}^{\delta}\right|+(1-1 /(C K))\left|s_{X}-s_{X^{\delta}}\right|,
$$

which implies

$$
\left|s_{X}-s_{X^{\delta}}\right| \leq C K \max _{s \leq \inf \left(s_{X}, s_{X^{\delta}}\right)}\left|X_{s}-X_{s}^{\delta}\right| .
$$

Using this estimate in (2.16), one concludes that

$$
\begin{aligned}
& \left|s_{X}-s_{X^{\delta}}\right| \leq C K \max _{s \leq \inf \left(s_{X}, s_{X^{\delta}}\right)}\left|X_{s}-X_{s}^{\delta}\right|,
\end{aligned}
$$

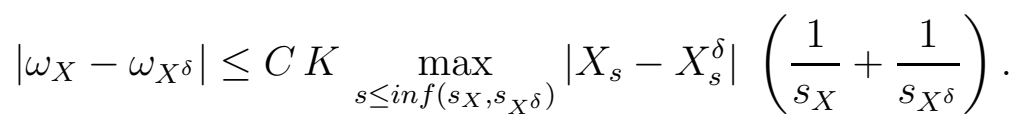

Inserting this in the term $I_{B m o d}$ enables to bound it by

$$
\begin{aligned}
I_{\text {Bmod }} \leq & C K^{k+2} \nu_{K} \int_{0}^{T} \int_{\Omega_{K}} \frac{\left|V(t, x, v)-V^{\delta}(t, x, v)\right|}{A_{\delta}(t, x, v)} \\
& \left(\int_{O_{X}^{t} \cap O_{X^{\delta}}^{t}}\left|G^{0}(z)\right| \max _{s \leq \inf \left(s_{X}, s_{X^{\delta}}\right)}\left|X_{s}-X_{s}^{\delta}\right|\left(\frac{1}{s_{X}^{3}}+\frac{1}{s_{X^{\delta}}^{3}}\right) d z\right. \\
& \left.+\int_{O_{X}^{t} \cap O_{X^{\delta}}^{t}}\left|G^{0}(z)\right|\left(\frac{\left|V_{s_{X}}-V_{s_{X}}^{\delta}\right|}{s_{X}^{2}}+\frac{\left|V_{s_{X} \delta}-V_{s_{X}^{\delta}}^{\delta}\right|}{s_{X^{\delta}}^{2}}\right) d z\right) d x d v d t,
\end{aligned}
$$


with $\nu_{K}=\max _{B(0, K)}|\nu(v)|$.

Changing back variables to $s, \omega$, one eventually finds

$$
\begin{aligned}
I_{\text {Bmod }} & \leq C K^{k+2} \nu_{K} \int_{0}^{T} \int_{\Omega_{K}} \frac{\left|V(t, x, v)-V^{\delta}(t, x, v)\right|}{A_{\delta}(t, x, v)} \\
& \left(\int_{0}^{t} \frac{\max _{r \leq s}\left|X_{s}-X_{s}^{\delta}\right|}{s} \int_{S^{2}}\left|G^{0}\left(X_{s}+s \omega\right)\right| d \omega d s\right. \\
& \left.+\int_{0}^{t} \int_{S^{2}}\left|G^{0}\left(X_{s}+s \omega\right)\right|\left|V_{s}-V_{s}^{\delta}\right| d \omega d s+\text { symmetric terms }\right) d x d v d t .
\end{aligned}
$$

Note that

$$
\max _{r \leq s}\left|X_{s}-X_{s}^{\delta}\right| \leq \sqrt{s}\left(\int_{0}^{s}\left|V_{r}-V_{r}^{\delta}\right|^{2} d r\right)^{1 / 2} \leq \sqrt{s} \sqrt{A_{\delta}(t, x, v)} .
$$

Hence by the definition of $A_{\delta}$

$$
\begin{aligned}
& \int_{0}^{T} \int_{\Omega_{K}} \frac{\left|V-V^{\delta}\right|(t, x, v)}{A_{\delta}(t, x, v)} \int_{0}^{t} \frac{\max _{r \leq s}\left|X_{s}-X_{s}^{\delta}\right|}{s} \int_{S^{2}}\left|G^{0}\left(X_{s}+s \omega\right)\right| d \omega d s \\
& \leq \int_{0}^{T} s^{-1 / 2} \int_{\Omega_{K}} \int_{S^{2}}\left|G^{0}\left(X_{s}+s \omega\right)\right| d \omega \int_{s}^{T} \frac{\left|V-V^{\delta}\right|(t, x, v)}{\sqrt{A_{\delta}(t, x, v)}} d t d x d v d s .
\end{aligned}
$$

However as $\left|V-V^{\delta}\right|^{2} \leq \partial_{t} A_{\delta}$

$$
\begin{aligned}
\int_{s}^{T} \frac{\left|V-V^{\delta}\right|(t, x, v)}{\sqrt{A_{\delta}(t, x, v)}} d t & \leq \sqrt{T}\left(\int_{s}^{T} \frac{\left|V-V^{\delta}\right|^{2}(t, x, v)}{A_{\delta}(t, x, v)} d t\right)^{1 / 2} \\
& \leq C \sqrt{-\log |\delta| \mid}
\end{aligned}
$$

one deduces that

$$
\begin{aligned}
& \int_{0}^{T} \int_{\Omega_{K}} \frac{\left|V-V^{\delta}\right|(t, x, v)}{A_{\delta}(t, x, v)} \int_{0}^{t} \frac{\max _{r \leq s}\left|X_{s}-X_{s}^{\delta}\right|}{s} \int_{S^{2}}\left|G^{0}\left(X_{s}+s \omega\right)\right| d \omega d s \\
& \leq C \sqrt{-\log |\delta| \mid} \int_{0}^{T} s^{-1 / 2} \int_{\Omega_{K}} \int_{S^{2}}\left|G^{0}\left(X_{s}+s \omega\right)\right| d \omega d x d v \\
& \leq C K^{3} \sqrt{-\log |\delta|}\left\|G^{0}\right\|_{L^{1}},
\end{aligned}
$$


by a change of variables.

Let us turn to the second term in(2.18). Denote

$$
\tilde{G}(s, x)=\int_{S^{2}}\left|G^{0}(x+s \omega)\right| d \omega
$$

By Cauchy-Schwartz, and since $A_{\delta}(t) \geq \int_{0}^{t}\left|V-V^{\delta}\right|^{2}$

$$
\begin{gathered}
\int_{0}^{T} \int_{\Omega_{K}} \frac{\left|V-V^{\delta}\right|(t, x, v)}{A_{\delta}(t, x, v)} \int_{0}^{t} \int_{S^{2}}\left|G^{0}\left(X_{s}+s \omega\right)\right|\left|V_{s}-V_{s}^{\delta}\right| d \omega d s d x d v d t \\
\quad \leq C \int_{\Omega_{K}}\left(\int_{0}^{T}\left|\tilde{G}\left(s, X_{s}\right)\right|^{2} d s\right)^{1 / 2} \int_{0}^{T} \frac{\left|V-V^{\delta}\right|(t, x, v) d t}{\sqrt{A_{\delta}(t, x, v)}} d x d v
\end{gathered}
$$

Hence

$$
\begin{aligned}
& \int_{0}^{T} \int_{\Omega_{K}} \frac{\left|V-V^{\delta}\right|(t, x, v)}{A_{\delta}(t, x, v)} \int_{0}^{t} \int_{S^{2}}\left|G^{0}\left(X_{s}+s \omega\right)\right|\left|V_{s}-V_{s}^{\delta}\right| d \omega d s d x d v d t \\
& \quad \leq C \sqrt{-\log |\delta|} \int_{\Omega_{K}}\left(\int_{0}^{T}\left|\tilde{G}\left(s, X_{s}\right)\right|^{2} d s\right)^{1 / 2} d x d v \\
& \quad \leq C|\Omega| \sqrt{-\log |\delta|} K^{3} \int_{0}^{T} \int_{\mathbb{R}^{3}}\left|\tilde{G}\left(s, X_{s}\right)\right|^{2} d x d s .
\end{aligned}
$$

By the usual estimates on solutions to wave equations (see for instance [23, chapter. 8, 5.21), one has

Lemma 2.3 For any $4 / 3<p<2$, there exists $C<\infty$ such that for all $G^{0} \in L^{p} \cap L^{1}$, we have

$$
\left\|\int_{S^{2}} G^{0}(x+s \omega) d \omega\right\|_{L^{2}} \leq C s^{3 / 2-3 / p}\left(\left\|G^{0}\right\|_{L^{1}}+\left\|G^{0}\right\|_{L^{p}}\right) .
$$

Combining all estimates and using Lemma 2.3 (note that $3-6 / p>-1$ if $p>4 / 3$ ), one finally obtains

$$
I_{B m o d} \leq C K^{5+k} \nu_{K} \sqrt{-\log |\delta|}\left(\left\|G^{0}\right\|_{L^{1}}+\left\|G^{0}\right\|_{L^{p}}\right) .
$$




\subsubsection{Bound on $I_{A}$}

Note that $O_{X}^{t} \backslash O_{X^{\delta}}^{t}=B\left(X_{t}, t\right) \backslash B\left(X_{t}^{\delta}, t\right)$. Since $\left|\dot{X}_{s}\right| \leq 1-1 / C K$ with the same for $X^{\delta}$, one has that for any $\omega \in S^{2}$

$$
\left|X_{t}^{\delta}-X_{s}-s \omega\right| \leq\left|X_{t}^{\delta}-x\right|+\left|X_{s}-x\right|+s \leq t(1-1 / C K)+2 s<t,
$$

if $s<t /(2 C K)$. Therefore

$$
\begin{gathered}
\forall z \in O_{X}^{t} \backslash O_{X^{\delta}}^{t}, \quad s_{X}(z) \geq \frac{t}{C K} \\
A \leq \frac{C K^{2}}{t^{2}} \int_{O_{X}^{t} \backslash O_{X^{\delta}}^{t}}\left|G^{0}(z)\right| d z+\frac{C K^{2}}{t^{2}} \int_{O_{X}^{t} \backslash O_{X^{\delta}}^{t}}\left|G^{0}(z)\right| d z .
\end{gathered}
$$

Now we introduce the following modified maximal operator

$$
\tilde{M} g(x)=\sup _{\eta \leq \varepsilon} \frac{1}{\varepsilon^{2} \eta} \int_{\varepsilon-\eta \leq|z-x| \leq \varepsilon}|g(z)| d z .
$$

Note that for example if $z \in O_{X}^{t} \backslash O_{X^{\delta}}^{t}$ then $\left|z-X_{t}\right| \leq t$ and $t \leq\left|z-X_{t}^{\delta}\right|$. Using that $\left|z-X_{t}^{\delta}\right| \leq\left|z-X_{t}\right|+\left|X_{t}-X_{t}^{\delta}\right|$, we deduce that

$$
t-\left|X_{t}-X_{t}^{\delta}\right| \leq\left|z-X_{t}\right| \leq t .
$$

Hence, one controls $A$ with $\tilde{M} G^{0}$ as

$$
A \leq C K^{2}\left|X_{t}-X_{t}^{\delta}\right|\left(\tilde{M} G^{0}\left(X_{t}\right)+\tilde{M} G^{0}\left(X_{t}^{\delta}\right)\right) .
$$

This allows for an easy bound on $I_{A}$ in terms of the $L^{1}$ norm of $\tilde{M} G^{0}$

$$
\begin{aligned}
I_{A} & =\int_{0}^{T} \int_{\Omega_{K}}\left(\nu\left(V_{t}\right)+\nu\left(V_{t}^{\delta}\right) \frac{\left|V_{t}-V_{t}^{\delta}\right|}{A_{\delta}(t, x, v)} A d x d v d t\right. \\
& \leq C K^{2} \nu(K) \int_{0}^{T} \int_{\Omega_{K}} \frac{\left|V_{t}-V_{t}^{\delta}\right|}{\sqrt{A_{\delta}(t, x, v)}}\left(\tilde{M} G^{0}\left(X_{t}\right)+\tilde{M} G^{0}\left(X_{t}^{\delta}\right)\right) d x d v d t \\
& \leq C K^{5} \nu(K) \sqrt{|\log \delta|}\left(\int_{|x| \leq C K}\left|\tilde{M} G^{0}(x)\right|^{2}\right)^{1 / 2},
\end{aligned}
$$

by the change of variable $(x, v) \rightarrow\left(X_{t}, V_{t}\right)$ (or $\left.\left(X_{t}^{\delta}, V_{t}^{\delta}\right)\right)$ and (2.19).

We hence need to estimate $\tilde{M} G^{0}$. As it is defined, it turns out that it is of the same order as the spherical maximal operator for which bounds are well known 
Lemma 2.4 $\forall p>3 / 2, \exists C>0$ s.t. for any smooth function $g$

$$
\|\tilde{M} g\|_{L^{p}\left(\mathbb{R}^{3}\right)} \leq C\|g\|_{L^{p}}
$$

Proof of Lemma 2.4. Simply note that

$$
\int_{\varepsilon-\eta \leq|z-x| \leq \varepsilon}|g(z)| d z \leq \int_{\varepsilon-\eta}^{\varepsilon} \int_{S(x, r)}|g(z)| d S(z) d r \leq C \eta \varepsilon^{2} M_{S} g(x),
$$

where $M_{S}$ is defined by

$$
M_{S} g(x)=\sup _{r} \oint_{S(x, r)}|g(z)| d S(z) .
$$

It is proved (see [23]) that in dimension $3, M_{S}$ is bounded on $L^{p}$ for any $p>3 / 2$ (the limit exponent is sharp) which easily implies the lemma.

Note that this exponent is also sharp for $\tilde{M}$ as obviously

$$
\sup _{\eta \leq \varepsilon} \frac{1}{\eta} \int_{\varepsilon-\eta \leq|z-x| \leq \varepsilon}|g(z)| d z \geq C \int_{S(x, \varepsilon)}|g(z)| d S(z) .
$$

And so $\tilde{M} g \geq C M_{S} g$.

Coming back to $I_{A}$ one concludes that

$$
I_{A} \leq C K^{8-3 / 2} \nu(K) \sqrt{|\log \delta|}\left\|G^{0}\right\|_{L^{2}} .
$$

\subsubsection{Bound for $I_{C}$}

Note that on $\partial B\left(X_{t}, t\right)$, one obviously has $s_{X}=t$ and $\omega_{X}=\left(z-X_{t}\right) / t$. Hence

$$
\int_{\partial B\left(X_{t}, t\right)} G^{0}(z) \frac{d S(z)}{s_{X}\left|\dot{X}_{s_{X}} \cdot \omega_{X}-1\right|}=C t \int_{S^{2}} \frac{G^{0}\left(X_{t}+t \omega\right)}{\left|\dot{X}_{t} \cdot \omega-1\right|} d \omega .
$$

One finds that

$$
\begin{aligned}
I_{C} & \leq C K^{2} \nu(K) \int_{\Omega_{K}} \int_{0}^{T} \frac{\left|V_{t}-V_{t}^{\delta}\right|^{2}}{A_{\delta}(t)} t \int_{S^{2}}\left|G^{0}\left(X_{t}+t \omega\right)\right| d \omega d x d v d t \\
+ & C \nu(K) \int_{\Omega_{K}} \int_{0}^{T} \frac{\left|V_{t}-V_{t}^{\delta}\right|}{A_{\delta}(t)} t\left|\int_{S^{2}} \frac{G^{0}\left(X_{t}+t \omega\right)-G^{0}\left(X_{t}^{\delta}+t \omega\right)}{\left|\dot{X}_{t}^{\delta} \cdot \omega-1\right|} d \omega\right| d x d v d t, \\
& =I_{C}^{1}+I_{C}^{2}
\end{aligned}
$$


Let us deal first with $I_{C}^{1}$. Noticing as before that

$$
\frac{\left|V_{t}-V_{t}^{\delta}\right|^{2}}{A_{\delta}(t)} \leq \partial_{t} \log A_{\delta}(t)
$$

Then denoting by $W$ the usual wave operator

$$
W g(t, x)=t \int_{S^{2}} g(x+t \omega) d \omega
$$

one finds, after integration by parts in time, that

$$
\begin{aligned}
I_{C}^{1} \leq & -C K^{2} \nu(K) \int_{0}^{T} \int_{\Omega_{K}} \log A_{\delta} \partial_{t}\left[W\left|G^{0}\right|\left(t, X_{t}\right)\right] \\
& +C K^{2} \int_{\Omega_{K}} \log A_{\delta}(T, x, v) W\left|G^{0}\right|\left(T, X_{T}\right) \\
\leq & C K^{13 / 2} \nu(K)|\log | \delta||\left(\left\|\partial_{t} W\left|G^{0}\right|\right\|_{L^{2}}+\left\|W\left|G^{0}\right|\right\|_{L^{2}}\right) .
\end{aligned}
$$

Of course $\left(\partial_{t}, \nabla_{x}\right) W\left|G^{0}\right|$ is bounded in $L^{2}$ by the norm of $G^{0}$ in $L^{2}$ as can be seen by taking the Fourier transform

$$
\hat{W} g(t, \xi)=\hat{g}(\xi) t \int_{S^{2}} e^{-i t \xi \cdot \omega} d \omega=\hat{g}(\xi) \frac{4 \pi \sin (|\xi| t)}{|\xi|} .
$$

Consequently

$$
I_{C}^{1} \leq C K^{13 / 2} \nu(K)|\log | \delta \mid\|\| G^{0} \|_{L^{2}} .
$$

Let us now turn to $I_{C}^{2}$. We have to define the modified wave operator

$$
\tilde{W}_{t} g(x, v)=t \int_{S^{2}} \frac{g(x+t \omega)}{\left|\frac{v}{\sqrt{1+v^{2}}} \cdot \omega-1\right|} d \omega .
$$

Note that $\tilde{W}_{t}$ enjoys the same regularizing properties as $W$. In particular

$$
\mathcal{F} \nabla_{v}^{k} \tilde{W}_{t} g(\xi, v)=\hat{g}(\xi) t \int_{S^{2}} \frac{\Phi_{k}(v, \omega) e^{-i t \xi \cdot \omega}}{\left|\frac{v}{\sqrt{1+v^{2}}} \cdot \omega-1\right|^{k+1}} d \omega
$$

for some smooth function $\Phi_{k}$ of $v$ and $\omega$. Therefore for any $k$

$$
\left\|\nabla_{x} \nabla_{v}^{k} \tilde{W}_{t} g\right\|_{L_{x v}^{2}} \leq C_{k} K^{k+7 / 2}\|g\|_{L^{2}}
$$


where the $L_{x v}^{2}$ norm is taken over any regular compact subset of $\mathbb{R}^{6}$ included in $B(0, K)$. By taking $k$ large enough $(k=2$ for instance) and by Sobolev embedding, one may conclude that

$$
\left\|\nabla_{x} \tilde{W}_{t} g\right\|_{L_{x}^{2}\left(L_{v}^{\infty}\right)} \leq C K^{15 / 2}\|g\|_{L^{2}}
$$

Notice now that

$$
\begin{aligned}
& \left|t \int_{S^{2}} \frac{G^{0}\left(X_{t}+t \omega\right)-G^{0}\left(X_{t}^{\delta}+t \omega\right)}{\left|\dot{X}_{t}^{\delta} \cdot \omega-1\right|} d \omega\right|=\left|\tilde{W}_{t} G^{0}\left(X_{t}, V_{t}^{\delta}\right)-\tilde{W}_{t} G^{0}\left(X_{t}^{\delta}, V_{t}^{\delta}\right)\right| \\
& \quad \leq\left|X_{t}-X_{t}^{\delta}\right|\left(M_{t}\left|\nabla_{x} \tilde{W}_{t} G^{0}\left(X_{t}^{\delta}, V_{t}^{\delta}\right)\right|+M_{t} \mid \nabla_{x} \tilde{W}_{t} G^{0}\left(X_{t}, V_{t}^{\delta}\right)\right),
\end{aligned}
$$

by applying Lemma 3.1 in [20], where we defined the modified maximal operator

$$
M_{s} g(x)=\frac{1}{|\delta|+\max _{r \leq s}\left|X_{r}-X_{r}^{\delta}\right|} \int_{B\left(x, \max _{r \leq s}\left|X_{r}-X_{r}^{\delta}\right|\right)} \frac{g(z) d z}{|z-x|^{2}},
$$

one has

$$
\begin{aligned}
I_{C}^{2} & \leq C \nu(K) \int_{\Omega_{K}} \int_{0}^{T} \frac{\left|V_{t}-V_{t}^{\delta}\right|}{\sqrt{A_{\delta}(t)}}\left(M_{t}\left|\nabla \tilde{W}_{t} G^{0}\left(X_{t}^{\delta}, V_{t}^{\delta}\right)\right|+M_{t}\left|\nabla \tilde{W}_{t} G^{0}\left(X_{t}, V_{t}^{\delta}\right)\right|\right) \\
& =I_{C}^{21}+I_{C}^{22} .
\end{aligned}
$$

The first term can be easily bounded as it is symmetric. By Cauchy-Schwarz, we have

$$
I_{C}^{2,1} \leq C \nu(K) K^{3} \sqrt{\log \delta}\left(\left(\int_{\Omega_{K}} \int_{0}^{T}\left(M_{t}\left|\nabla_{x} \tilde{W}_{t} G^{0}\left(X_{t}^{\delta}, V_{t}^{\delta}\right)\right|\right)^{2} d t d x d v\right)^{1 / 2}\right.
$$

Now by Fubini and a change of variable

$$
\begin{aligned}
\int_{\Omega_{K}} \int_{0}^{T}( & \left(M_{t}\left|\nabla_{x} \tilde{W}_{t} G^{0}\left(X_{t}^{\delta}, V_{t}^{\delta}\right)\right|\right)^{2} d t d x d v \\
& \leq \int_{0}^{T} \int_{B(0, C K)}\left(M_{t}\left|\nabla W_{t} G^{0}(x, v)\right|\right)^{2} d x d v d t \\
& \leq C \int_{0}^{T} \int_{B(0, C K)}\left|\nabla_{x} \tilde{W}_{t} G^{0}(x, v)\right|^{2} d x d v d t
\end{aligned}
$$


by the continuity of $M_{t}$ on $L^{2}$. By the bound (2.24), one may bound

$$
I_{C}^{2,1} \leq C \nu(K) K^{11 / 2}\left\|G^{0}\right\|_{L^{2}}
$$

The other term is not symmetric, as it mixes $X_{t}^{\delta}$ and $V_{t}$. It is hence more complicated but it can still be handled in a roughly similar way

$$
I_{C}^{2,2} \leq C \nu(K) K^{3} \sqrt{|\log \delta|}\left(\int_{0}^{T} \int_{\Omega_{K}} M_{t}\left(\left|\nabla_{x} \tilde{W} G^{0}\left(X_{t}, V_{t}^{\delta}\right)\right|^{2}\right) d x d v d t\right)^{1 / 2} .
$$

Now note that

$$
M_{t} g(x) \leq \int_{B(0, K)} \frac{|g(z)| d z}{(|\delta|+|z-x|)|z-x|^{2}}
$$

Hence one gets

$$
\begin{aligned}
I_{C}^{2,2} \leq & C \nu(K) K^{3}|\log \delta|^{1 / 2} \\
& \left(\int_{0}^{T} \int_{(B(0, K))^{3}} \frac{\left|\nabla_{x} \tilde{W} G^{0}\left(z, V_{t}^{\delta}\right)\right|^{2}}{\left(|\delta|+\left|z-X_{t}\right|\right)\left|z-X_{t}\right|^{2}} d z d x d v d s\right)^{1 / 2} .
\end{aligned}
$$

Therefore

$$
\begin{aligned}
I_{C}^{2,2} & \leq C \nu(K) K^{3}|\log \delta|^{1 / 2} \\
& \left(\int_{0}^{T} \int_{(B(0, K))^{3}} \frac{\sup _{w}\left|\nabla_{x} \tilde{W} G^{0}(z, w)\right|^{2}}{\left(|\delta|+\left|z-X_{t}\right|\right)\left|z-X_{t}\right|^{2}} d z d x d v d s\right)^{1 / 2} .
\end{aligned}
$$

Now by the usual change of variables, we get

$$
I_{C}^{2,2} \leq C \nu(K) K^{9 / 2}|\log \delta|\left\|\nabla_{x} \tilde{W} G^{0}\right\|_{L_{x}^{2}\left(L_{v}^{\infty}\right)} .
$$

Using (2.25), one deduces that

$$
I_{C}^{2,2} \leq C \nu(K) K^{12}|\log \delta|\left\|G^{0}\right\|_{L^{2}}
$$

Therefore, combining with (2.22) and (2.26), one finally concludes that

$$
I_{C} \leq C K^{12} \nu(K)|\log \delta|\left\|G^{0}\right\|_{L^{2}} .
$$

We did not deal with this term in a very subtle manner here. However to improve the result, one would need to do $L^{1}$ or at least $L^{p}$ bounds (instead of $L^{2}$ ). Note as well that other terms anyway impose the use of the $L^{2}$ norm. 


\subsubsection{Bound for $I_{D}$}

This bound essentially follows the line of $I_{A}$ in a slightly more complicated way.

First of all, one may easily compute the $z$-derivative as

$$
\nabla_{z} s_{X}=\frac{\omega_{X}}{\omega_{X} \cdot \dot{X}_{s_{X}}-1}, \quad \nabla_{z} \omega_{X}=\frac{1}{s_{X}}\left(\frac{\omega_{X} \otimes\left(\dot{X}_{s_{X}}-\omega\right)}{\omega_{X} \cdot \dot{X}_{s_{X}}-1}-I\right)
$$

and the corresponding formulas for $s_{X^{\delta}}$ and $\omega_{X^{\delta}}$. Hence

$$
\begin{aligned}
\left|\nabla_{z} \cdot \frac{C \omega_{X}}{s_{X}\left|\dot{X}_{s_{X}} \cdot \omega_{X}-1\right|}\right| & \leq \frac{C\left|1+\dot{V}_{s_{X}}\right|}{s_{X}^{2}\left|\dot{X}_{s_{X}} \cdot \omega_{X}-1\right|^{2}} \\
& \leq \frac{C\left|1+F\left(s_{X}, X_{s_{X}}\right)\right|}{s_{X}^{2}\left|\dot{X}_{s_{X}} \cdot \omega_{X}-1\right|^{2}}
\end{aligned}
$$

Inserting the corresponding terms in $I_{D}$, one finds that

$$
\begin{aligned}
I_{D} \leq & C K^{2} \nu(K) \int_{\Omega_{K}} \int_{0}^{T} \frac{\left|V_{t}-V_{t}^{\delta}\right|}{A_{\delta}(t)} \int_{O_{X}^{t} \backslash O_{X^{\delta}}^{t}} \frac{\left|G^{0}(z)\right|}{s_{X}^{2}}\left|1+F\left(s_{X}, X_{s_{X}}\right)\right| \\
& + \text { symmetric term. }
\end{aligned}
$$

The only difference with $I_{A}$ is the additional term $F\left(s_{X}, X_{s_{X}}\right)$. Now, note that as before

$$
O_{X}^{t} \backslash O_{X^{\delta}}^{t} \subset\left\{t-\left|X_{t}-X_{t}^{\delta}\right| \leq\left|z-X_{t}\right| \leq t\right\}
$$

Hence, using spherical coordinates, one gets

$$
\begin{aligned}
I_{D} \leq & C K^{2} \nu(K) \int_{\Omega_{K}} \int_{0}^{T} \frac{\left|V_{t}-V_{t}^{\delta}\right|}{A_{\delta}(t)} \int_{t-\left|X_{t}-X_{t}^{\delta}\right|}^{t}\left|1+F\left(s, X_{s}\right)\right| \\
& \int_{S^{2}}\left|G^{0}\left(X_{t}+s \omega\right)\right| d \omega d s d t d x d v+\text { symmetric } \\
\leq & C K^{2} \nu(K) \int_{\Omega_{K}} \int_{0}^{T} \frac{\left|V_{t}-V_{t}^{\delta}\right|}{A_{\delta}(t)} M_{S} G^{0}\left(X_{t}\right) \int_{t-\left|X_{t}-X_{t}^{\delta}\right|}^{t}\left|1+F\left(s, X_{s}\right)\right| d s \\
& + \text { symmetric, }
\end{aligned}
$$


with $M_{S}$ as before the spherical maximal function. By Cauchy-Schwartz

$$
\begin{aligned}
I_{D} \leq & C K^{2} \nu(K)\left(\int_{\Omega_{K}} \int_{0}^{T} \frac{\left|V_{t}-V_{t}^{\delta}\right|^{2}}{A_{\delta}(t)}\left(M_{S} G^{0}\left(X_{t}\right)\right)^{2} d t d x d v\right. \\
& \left.\int_{\Omega_{K}} \int_{0}^{T} \frac{\left|X_{t}-X_{t}^{\delta}\right|}{A_{\delta}(t)} \int_{t-\left|X_{t}-X_{t}^{\delta}\right|}^{t}\left(1+F\left(s, X_{s}\right)\right)^{2} d s d t d x d v\right)^{1 / 2} .
\end{aligned}
$$

The second term is bounded, using Fubini and then changing variables to $(x, v)$ from $\left(X_{s}, V_{s}\right)$, and is less than

$$
C K^{6}\|F\|_{L^{2}}^{2} \leq C K^{6} T\left\|F^{0}\right\|_{L^{2}}^{2},
$$

as the wave operator propagates the $L^{2}$ norm.

As for the first term, change variables to $(x, v)$ from $\left(X_{t}, V_{t}\right)$ to bound it by

$$
\int_{B(0, K)}\left(M_{S} G^{0}(x)\right)^{2} \int_{0}^{T} \frac{\left|v-V_{t}^{\delta}\left(V_{t}^{-1}(x, v)\right)\right|^{2}}{\delta^{2}+\int_{0}^{t}\left|V_{s}\left(V_{t}^{-1}(x, v)\right)-V_{s}^{\delta}\left(V_{t}^{-1}(x, v)\right)\right|^{2} d s} d t d x d v
$$

Note that by the semi-group property, one still has that

$$
\left|v-V_{t}^{\delta}\left(V_{t}^{-1}(x, v)\right)\right|^{2}=\partial_{t} \int_{0}^{t}\left|V_{s}\left(V_{t}^{-1}(x, v)\right)-V_{s}^{\delta}\left(V_{t}^{-1}(x, v)\right)\right|^{2} d s,
$$

and hence the previous term is bounded by

$$
\sqrt{-\log |\delta|} \int_{B(0, K)}\left(M_{S} G^{0}(x)\right)^{2} d x d v \leq C K^{3} \sqrt{-\log |\delta|}\left\|G^{0}\right\|_{L^{2}}
$$

as the spherical maximal function is bounded on $L^{2}$.

Combining all the estimates, one eventually finds that

$$
I_{D} \leq C K^{11 / 2} \nu(K) \sqrt{|\log \delta|}\left\|G^{0}\right\|_{L^{2}}\left\|F^{0}\right\|_{L^{2}} .
$$

Like $I_{C}$ this term requires the $L^{2}$ norm of $G^{0}$. Contrary to $I_{C}$ though, the computation here naturally produces a quadratic term in $G^{0}$ and $F^{0}$ and one does not see very well how to improve on it. 


\subsubsection{Bound for $I_{E}$}

Applying formula (2.28), and recalling that $\phi \leq 1$, we can decompose $E$ into

$$
\begin{gathered}
|E| \leq C \int_{O_{X}^{t} \cap O_{X^{\delta}}^{t}}\left|G^{0}(z)\right|\left|\frac{H\left(s_{X}, \omega_{X}\right)}{s_{X}^{2}\left|\dot{X}_{s_{X}} \cdot \omega_{X}-1\right|^{2}}-\frac{H\left(s_{X^{\delta}}, \omega_{X^{\delta}}\right)}{s_{X^{\delta}}^{2}\left|\dot{X}_{s_{X^{\delta}}} \cdot \omega_{X^{\delta}}-1\right|^{2}}\right| d z \\
+\mid \int_{O_{X}^{t} \cap O_{X^{\delta}}^{t}} G^{0}(z)\left(\frac{\dot{V}_{s_{X}} \cdot \omega_{X}}{s_{X}\left(\dot{X}_{s_{X}} \cdot \omega_{X}-1\right)^{3}}\right. \\
\left.-\frac{\dot{V}_{s_{X^{\delta}}} \cdot \omega_{X^{\delta}}}{s_{X^{\delta}}\left(\dot{X}_{s_{X^{\delta}}} \cdot \omega_{X^{\delta}}-1\right)^{3}}\right) d z \mid=E^{1}+E^{2} .
\end{gathered}
$$

for some explicit smooth function $H$ whose exact expression is unimportant here.

The term $I_{E}^{1}$ is bounded by a direct application of Lemma 2.2

$$
I_{E}^{1} \leq C \nu(K) K^{7} \sqrt{-\log |\delta|}\left(\left\|G^{0}\right\|_{L^{1}}+\left\|G^{0}\right\|_{L^{p}}\right)
$$

As for $E^{2}$, we change back variables to find

$$
\begin{gathered}
E^{2} \leq\left|\int_{0}^{t}\left(\dot{V}_{s}-\dot{V}_{s}^{\delta}\right) \phi(s) d s\right|+\mid \int_{0}^{t} \dot{V}_{s} s \int_{S^{2}}\left(\frac{\omega G^{0}\left(X_{s}+s \omega\right)}{\left(1-\dot{X}_{s} \cdot \omega\right)^{2}}\right. \\
\left.-\frac{\omega G^{0}\left(X_{s}^{\delta}+s \omega\right)}{\left(1-\dot{X}_{s}^{\delta} \cdot \omega\right)^{2}}\right) d \omega \mid=E^{2,1}+E^{2,2},
\end{gathered}
$$

with

$$
\phi(s)=s \int_{S^{2}} G^{0}\left(X_{s}+s \omega\right) \frac{\omega}{\left(1-\dot{X}_{s} \cdot \omega\right)^{2}} d \omega .
$$

The term $E^{2,2}$ is treated in a similar manner as the previous ones (note in particular that $\dot{V}_{s}$ is bounded in $L^{2}$ by $\left\|F^{0}\right\|_{L^{2}}$ ). We instead focus on $E^{2,1}$ and remark that by integration by part

$$
\begin{aligned}
I_{E}^{2,1} \leq & \int_{\Omega_{K}} \int_{0}^{T} \frac{\left|V_{t}-V_{t}^{\delta}\right|^{2}}{A_{\delta}(t)}|\phi(t, x, v)| d x d v d t \\
& +\int_{\Omega_{K}} \int_{0}^{T} \frac{\left|V_{t}-V_{t}^{\delta}\right|}{A_{\delta}(t)} \int_{0}^{t}\left|V_{s}-V_{s}^{\delta}\right|\left|\partial_{s} \phi(s, x, v)\right| d s d t d x d v .
\end{aligned}
$$


Note that

$$
\begin{aligned}
\int_{\Omega_{K}} \int_{0}^{T} \frac{\left|V_{t}-V_{t}^{\delta}\right|^{2}}{A_{\delta}(t)}|\phi(t, x, v)| d x d v d t \\
\quad \leq C K^{2} \int_{\Omega_{K}} \int_{0}^{T} \partial_{t} \log \left(A_{\delta}(t) \mid\right) t \int_{S^{2}}\left|G^{0}\left(X_{t}+t \omega\right)\right| d \omega \\
\quad \leq C K^{2}|\log | \delta|| \int_{\Omega_{K}} \int_{0}^{T}\left|\partial_{t}\left(t \int_{S^{2}}\left|G^{0}\left(X_{t}+t \omega\right)\right| d \omega\right)\right| d t d x d v
\end{aligned}
$$

We remark that

$$
\begin{aligned}
\left|\partial_{t}\left(t \int_{S^{2}} g\left(X_{t}+t \omega\right) d \omega\right)\right| \leq & C K\left|t \int_{S^{2}}(1, \omega) \nabla g\left(X_{t}+t \omega\right) d \omega\right| \\
& +\int_{S^{2}}\left|g\left(X_{t}+t \omega\right)\right| d \omega
\end{aligned}
$$

which implies that this term is bounded in $L^{2}$ by the $L^{2}$ norm of $g$. Consequently

$$
\int_{\Omega_{K}} \int_{0}^{T} \frac{\left|V_{t}-V_{t}^{\delta}\right|^{2}}{A_{\delta}(t)}|\phi(t, x, v)| d x d v d t \leq C K^{2}|\log | \delta\|\| G^{0} \|_{L^{2}} .
$$

As for the other term in $E^{2,1}$, compute

$$
\begin{aligned}
\left|\partial_{s} \phi\right| & \leq \int_{S^{2}} \frac{\left|G^{0}\left(X_{s}+s \omega\right)\right|}{\left(1-\dot{X}_{s} \cdot \omega\right)^{2}}+s\left|\int_{S^{2}} \frac{\omega \cdot \nabla G^{0}\left(X_{s}+s \omega\right) \omega}{\left(1-\dot{X}_{s} \cdot \omega\right)^{2}} d s\right| \\
& +C K^{3}\left|\dot{V}_{s}\right| \int_{S^{2}}\left|G^{0}\left(X_{s}+s \omega\right)\right| d s .
\end{aligned}
$$

The first two terms are treated similarly as before. For the last term note that by Cauchy-Schwartz

$$
\begin{aligned}
\int_{\Omega_{K}} \int_{0}^{T} & \frac{\left|V_{t}-V_{t}^{\delta}\right|}{A_{\delta}(t)} \int_{0}^{t}\left|V_{s}-V_{s}^{\delta}\right|\left|\dot{V}_{s}\right| \int_{S^{2}}\left|G^{0}\left(X_{s}+s \omega\right)\right| d s d t d x d v \\
\leq & \left(\int_{\Omega_{K}} \int_{0}^{T} \int_{0}^{t}\left|\dot{V}_{s}\right|^{2} \frac{\left|V_{t}-V_{t}^{\delta}\right|^{2}}{A_{\delta}(t)} d s d t d x d v\right)^{1 / 2} \\
& \left(\int_{\Omega_{K}} \int_{0}^{T}\left|M_{S} G^{0}\left(X_{s}\right)\right|^{2} \frac{\int_{0}^{t}\left|V_{s}-V_{s}^{\delta}\right|^{2} d s}{A_{\delta}(t)} d s d t d x d v\right)^{1 / 2}
\end{aligned}
$$


with as always $M_{S}$ the spherical maximal operator. Since $G^{0} \in L^{2}, M_{S}$ is bounded on $L^{2}$ and $\dot{V}_{s}$ is also bounded in $L^{2}$ by the $L^{2}$ norm of $G^{0}$,

$$
\begin{aligned}
& \int_{\Omega_{K}} \int_{0}^{T} \frac{\left|V_{t}-V_{t}^{\delta}\right|}{A_{\delta}(t)} \int_{0}^{t}\left|V_{s}-V_{s}^{\delta}\right|\left|\dot{V}_{s}\right| \int_{S^{2}}\left|G^{0}\left(X_{s}+s \omega\right)\right| d s d t d x d v \\
& \quad \leq C K^{3} \sqrt{-\log |\delta|}\left\|G^{0}\right\|_{L^{2}}^{2} .
\end{aligned}
$$

We conclude that

$$
I_{E} \leq C \nu(K) K^{7} \sqrt{-\log |\delta|}\left(\left\|G^{0}\right\|_{L^{1}}+\left\|G^{0}\right\|_{L^{2}}+\left\|G^{0}\right\|_{L^{2}}\left\|F^{0}\right\|_{L^{2}}\right) .
$$

\subsubsection{Conclusion of the proof of Lemma 2.1}

We combine estimates (2.15), (2.21), (2.27), (2.29) and (2.31). Taking the worst terms, one finds the estimate in Lemma 2.1.

\subsection{Conclusion of the proof of Proposition 2.1}

By Lemma 2.1, one has that

$$
\bar{Q}_{\delta}(T) \leq C_{K} T|\log | \delta \|\left(\left\|G^{0}\right\|_{L^{1}}+\left\|G^{0}\right\|_{L^{2}}\right)\left(1+\left\|F^{0}\right\|_{L^{2}}\right),
$$

for a constant $C_{K}$ increasing with $K$ (which could be made explicit for a given $\nu$ ).

On the other hand, the definition of $\bar{Q}_{\delta}$ yields the very obvious bound

$$
\bar{Q}_{\delta}(T) \leq C_{K} T \sqrt{-\log |\delta|}\left\|G^{0}\right\|_{W^{1, \infty}} .
$$

As $\bar{Q}_{\delta}(T)$ depends linearly on $G^{0}$, one may conclude by interpolation that for any fixed $G^{0} \in L^{2}$, there exists an increasing $\psi_{0}$ with

$$
\frac{\psi_{0}(\xi)}{\xi} \longrightarrow 0, \quad \text { as } \xi \rightarrow \infty
$$

such that

$$
\bar{Q}_{\delta}(T) \leq C_{K} T \psi_{0}(-\log |\delta|)
$$

Note that $\psi_{0}$ depends on $\left\|G^{0}\right\|_{L^{2}}$ and on the equi-integrability of $\hat{G}^{0}$ in $L^{2}$. As $F^{0} \in L^{2}$, we finally use this estimate for $G^{0}=F^{0}$ and get

$$
Q_{\delta}(T) \leq \frac{C T}{K^{2}}|\log | \delta||+C_{K} T \psi_{0}(-\log |\delta|)
$$


It only remains to optimize in $K$ by defining

$$
\psi(\xi)=\inf _{K}\left(\frac{C \xi}{K^{2}}+C_{K} \psi_{0}(\xi)\right) .
$$

One still has that

$$
\frac{\psi(\xi)}{\xi} \longrightarrow 0, \quad \text { as } \xi \rightarrow \infty
$$

and

$$
Q_{\delta}(T) \leq T \psi(-\log |\delta|)
$$

which concludes the proof of Prop. 2.1.

\section{Proof of Theorem 1.2}

\subsection{Well posedness for the corresponding ODE}

We follow the same steps as for the proof of Th. 1.1. We study the ODE

$$
\begin{aligned}
& \frac{d}{d t} X(t, x, v)=\alpha(V(t, x, v)), \quad \frac{d}{d t} V(t, x, v)=F(t, X(t, x, v), \\
& X(0, x, v)=x, \quad V(0, x, v)=v .
\end{aligned}
$$

As flows the solutions are again required to satisfy

Property 2 For any $t \in \mathbb{R}$ the application

$$
(x, v) \in \mathbb{R} \times \mathbb{R}^{d} \mapsto(X(t, x, v), V(t, x, v)) \in \mathbb{R} \times \mathbb{R}^{d}
$$

is globally invertible and has Jacobian 1 at any $(x, v) \in \mathbb{R} \times \mathbb{R}^{d}$. It also defines a semi-group

$$
\begin{array}{ll}
\forall s, t \in \mathbb{R}, & X(t+s, x, v)=X(s, X(t, x, v), V(t, x, v)), \\
\text { and } & V(t+s, x, v)=V(s, X(t, x, v), V(t, x, v)) .
\end{array}
$$

We look again at

$$
\begin{aligned}
R_{\delta}(T)=\log \left(1+\frac{1}{\bar{\delta}(T, x, v,|\delta|)^{2}}\left(\sup _{0 \leq t \leq T}\left|X(t, x, v)-X^{\delta}(t, x, v)\right|^{2}\right.\right. \\
\left.\left.\quad+\int_{0}^{T}\left|V(t, x, v)-V^{\delta}(t, x, v)\right|^{2} d t\right)\right)
\end{aligned}
$$


for any $(x, v) \in \Omega$ a subset of $\mathbb{R}^{d+1}$, and for a function $\bar{\delta}(t, x, v)=\bar{\delta}\left(t, x, v,|\delta|^{2}\right)$ to be determined later. $(X, V)$ is a solution to (3.1) satisfying Property 2 (or a regularized problem) and $X^{\delta}, V^{\delta}$ verifies

Either $\left(X^{\delta}, V^{\delta}\right)$ is a solution to (3.1) (or a regularized version)

$$
\begin{gathered}
\text { satisfying Property } 2, \\
\text { Or } \exists\left(\delta_{1}, \delta_{2}\right) \in \mathbb{R}^{1+d} \quad \text { with }\left|\left(\delta_{1}, \delta_{2}\right)\right| \leq \delta, \\
\left(X^{\delta}, V^{\delta}\right)(t, x, v)=(X, V)\left(t, x+\delta_{1}, v+\delta_{2}\right) .
\end{gathered}
$$

Theorem 1.2 is a consequence of

Proposition 3.1 Assume $F^{0} \in L^{\infty}$ and (1.6). For any $\Omega$ compact, any $F^{0}$ bounded, there exists a function $\bar{\delta}(t, x, v,|\delta|)$, increasing in time with $\bar{\delta}(0, x, v,|\delta|)=|\delta|$, such that for any $(X, V)$ solution to (3.1) with $F$ given by Eq. (1.5), satisfying Property 2 , and $\left(X^{\delta}, V^{\delta}\right)$ satisfying (3.4), for all $T>0$,

$$
R_{\delta}(T) \leq C T\left\|F^{0}\right\|_{\infty}(\log (1 /|\delta|))^{3 / 4}, \bar{\delta}(T, x, v,|\delta|) \longrightarrow 0 \text { as }|\delta| \rightarrow 0 \text {, a.e. }(x, v) .
$$

First of all let us order the $\mu_{n}$ decreasingly

$$
\left|\mu_{0}\right| \geq\left|\mu_{1}\right| \geq \ldots\left|\mu_{n}\right| \ldots
$$

Note then that by (1.6)

$$
\|F(t, x)\|_{\infty} \leq\left\|F_{0}\right\|_{\infty} \sum_{n}\left|\alpha_{n}\right| \leq C\left\|F^{0}\right\|_{\infty} .
$$

Therefore defining $\tilde{\Omega}=\Omega+B\left(0, T\left\|F_{0}\right\|_{\infty} \sum_{n}\left|\alpha_{n}\right|\right)$, one has that $(X, V) \in \tilde{\Omega}$ for any $(x, v) \in \Omega$ and $t \in[0, T]$. The same is of course true for $\left(X^{\delta}, V^{\delta}\right)$. As before problems occur when the velocity of the particle is close to one of the propagation velocities $\xi_{n}$. So first of all it is necessary to control the time that each trajectory spends near one of those points.

Denote

$$
\begin{aligned}
& \omega(w, \eta, K)=\{(x, v) \in \Omega \text { s.t. }|\{t,|\alpha(V(t, x, v))-w| \leq \eta\}| \geq K \eta\}, \\
& \omega^{\delta}(w, \eta, K)=\left\{(x, v) \in \Omega \text { s.t. }\left|\left\{t,\left|\alpha\left(V^{\delta}\right)(t, x, v)-w\right| \leq \eta\right\}\right| \geq K \eta\right\} .
\end{aligned}
$$

The parameter $\eta$ will be chosen later but will tend to 0 as $|\delta| \rightarrow 0$. Then 
Lemma 3.1 There exists a constant $C$ depending on $\left\|F^{0}\right\|_{\infty}$ and $\sum_{n}\left|\mu_{n}\right|$ such that

$$
|\omega(w, \eta, K)| \leq \frac{C}{K}
$$

Proof. Simply write that

$$
\int_{0}^{T} \int_{\omega(w, \eta, K)} \mathbb{I}_{\{|\alpha(V(t, x, v))-w| \leq \eta\}} d x d v d t \geq K \eta|\omega(w, \eta, K)| .
$$

On the other hand, using Property 3.2 and the assumption (1.4) on $\alpha(v)$, we have

$$
\begin{aligned}
\int_{0}^{T} \int_{\omega(w, \eta, K)} \mathbb{I}_{\{|\alpha(V(t, x, v))-w| \leq \eta\}} d x d v d t & \leq \int_{0}^{T} \int_{\tilde{\Omega}} \mathbb{I}_{\{|\alpha(v)-w| \leq \eta\}} d x d v d t \\
& \leq C|\tilde{\Omega}| \eta T
\end{aligned}
$$

for some constant $C$.

Finally one concludes that

$$
|\omega(w, \eta, K)| \leq C / K
$$

For $X^{\delta}, V^{\delta}$, one uses (3.4) and either they also satisfy (3.2) in which case the proof is identical. Or one just has to shift $(x, v)$ by $\delta$ and still follow the same steps.

Now for $a_{n}$ (to be fixed later), we define

$$
O_{n}=\left\{v,\left|\alpha(v)-\xi_{n}\right| \leq a_{n} \eta\right\} .
$$

Then for any $(x, v)$, we decompose the time interval $[0, T]$ into $I_{x, v}$ and the union $\bigcup_{n}\left[t_{0}^{n}, s_{0}^{n}\right] \cup \ldots \cup\left[t_{k_{n}}^{n}, s_{k_{n}}^{n}\right]$ with

$$
\begin{aligned}
& I_{x, v}=\left\{t,\left|\alpha(V(t, x, v))-\xi_{n}\right| \geq a_{n} \eta / 2\right\}, \\
& \sup _{\left[t_{i}^{n}, s_{i}^{n}\right]}\left|\alpha(V)-\xi_{n}\right|=a_{n} \eta, \inf _{\left[t_{i}^{n}, s_{i}^{n}\right]}\left|\alpha(V)-\xi_{n}\right| \leq a_{n} \eta / 2 .
\end{aligned}
$$

Similarly one defines $I_{x, v}^{\delta}$ and the intervals $\left[t_{i}^{n, \delta}, s_{i}^{n, \delta}\right]$ for $i=0 \ldots k_{n}^{\delta}$. Note that $k_{n}$ and $k_{n}^{\delta}$ depend on $(x, v)$. 
Define now

$$
l_{n}=\sum_{i=0}^{k_{n}}\left(s_{i}^{n}-t_{i}^{n}\right), \quad l_{n}^{\delta}=\sum_{i=0}^{k_{n}}\left(s_{i}^{n, \delta}-t_{i}^{n, \delta}\right),
$$

and

$$
\begin{aligned}
& \omega(\eta, K)=\left\{(x, v) \in \Omega \text { s.t. } \sum_{n} l_{n} \geq K \eta\right\}, \\
& \omega^{\delta}(\eta, K)=\left\{(x, v) \in \Omega \text { s.t. } \sum_{n} l_{n}^{\delta} \geq K \eta\right\} .
\end{aligned}
$$

Similarly to Lemma 3.1 one can deduce

Lemma 3.2 Assume that $\sum_{n} a_{n}<\infty$, then there exists a constant $C$ depending on $\sum_{n} a_{n}$ s.t.

$$
|\omega(\eta, K)| \leq \frac{C}{K}, \quad\left|\omega^{\delta}(\eta, K)\right| \leq \frac{C}{K}
$$

Proof. Simply note that

$$
\omega(\eta, K) \subset\left\{(x, v), \quad\left|\left\{t, V(t, x, v) \in \bigcup_{n} O_{n}\right\}\right| \geq K \eta\right\} .
$$

Then similarly to the proof of Lemma 3.1

$$
\begin{aligned}
K \eta|\omega(\eta, K)| & \leq \int_{\omega(\eta, K)} \int_{0}^{T} \mathbb{I}_{V(t, x, v) \in \bigcup_{n} O_{n}} d t d x d v \\
& \leq \int_{0}^{T} \int_{\Omega} \mathbb{I}_{V(t, x, v) \in \cup_{n} O_{n}} d x d v d t=\int_{0}^{T} \int_{\tilde{\Omega}} \mathbb{I}_{v \in \cup_{n} O_{n}} d x d v \\
& \leq T \sum_{n}\left|O_{n}\right| \leq T \eta \sum a_{n},
\end{aligned}
$$

which shows the result.

We are now ready to define $\bar{\delta}(t, x, v,|\delta|)$. We put

$$
\bar{\delta}(0, x, v,|\delta|)=|\delta|, \quad \partial_{t} \bar{\delta}(0, x, v,|\delta|)=C\|F\|_{\infty} \sum_{n}\left(l_{n}+l_{n}^{\delta}\right) .
$$

Note that from Lemma 3.2 and the fact that $\eta$ tends to 0 as $|\delta| \rightarrow 0, \bar{\delta}$ indeed converges to 0 for a.e. $(x, v)$. 
From the computation for $Q_{\delta}$ in the proof of Proposition 2.1, one sees that

$$
\begin{aligned}
\frac{d}{d t} R_{\delta}(T) \leq & 2 \int_{0}^{T} \frac{V(t)-V^{\delta}(t)}{A_{\delta}(t, x, v)} \\
& \int_{0}^{t}\left(F(s, X(s, x, v))-F\left(s, X^{\delta}(s, x, v)\right)\right) d s d t \\
& -2 \int_{0}^{T} \frac{\partial_{t} \bar{\delta}(t, x, v,|\delta|)}{A_{\delta}(t, x, v)} d t
\end{aligned}
$$

with

$$
\begin{aligned}
A_{\delta}(t, x, v)= & \bar{\delta}^{2}+\sup _{0 \leq s \leq t}\left|X(s, x, v)-X^{\delta}(s, x, v)\right|^{2} \\
& +\int_{0}^{t}\left|V(s, x, v)-V^{\delta}(s, x, v)\right|^{2} d s
\end{aligned}
$$

Compute

$$
\begin{aligned}
\int_{0}^{t}(F & \left.(s, X(s))-F\left(s, X^{\delta}(s)\right)\right) d s \\
= & \sum_{n} \int_{0}^{t}\left(F^{0}\left(X(s)-\xi_{n} s\right)-F^{0}\left(X^{\delta}(s)-\xi_{n} s\right)\right) \mu_{n} d s \\
\leq \sum_{n} & \left(C\|F\|_{\infty} \mu_{n} \sum_{i=0}^{k_{n}}\left|s_{i}^{n}-t_{i}^{n}\right|+C\|F\|_{\infty} \mu_{n} \sum_{i=0}^{k_{n}^{\delta}}\left|s_{i}^{n, \delta}-t_{i}^{n, \delta}\right|\right. \\
& \left.\quad+\int_{s \notin \cup_{i}\left(\left[t_{i}^{n}, s_{i}^{n}\right] \cup\left[t_{i}^{n, \delta}, s_{i}^{n, \delta}\right]\right)}\left(F^{0}\left(X(s)-\xi_{n} s\right)-F^{0}\left(X^{\delta}(s)-\xi_{n} s\right)\right) \mu_{n} d s\right) .
\end{aligned}
$$

Now $[0, T] \backslash \cup_{i}\left[t_{i}^{n}, s_{i}^{n}\right] \cup\left[t_{i}^{n, \delta}, s_{i}^{n, \delta}\right]$ is included in an union of intervals of the form $\left[s_{i}^{n}, t_{i+1}^{n}\right],\left[s_{i}^{n, \delta}, t_{i+1}^{n, \delta}\right],\left[s_{i}^{n}, t_{j}^{n \delta}\right]$ or $\left[s_{i}^{n, \delta}, t_{j}^{n}\right]$. This depends only on whether $V(s)$ or $V^{\delta}(s)$ is the first or the last to be such that $\left|\alpha(V)-\xi_{n}\right|=a_{n} \eta$. Assume for instance that the corresponding interval is $\left[s_{i}^{n}, t_{i+1}^{n}\right]$.

Define the transforms

$$
\Phi_{n}(s)=X(s)-\xi_{n} s, \quad \Phi_{n}^{\delta}(s)=X^{\delta}(s)-\xi_{n} s
$$

and note that by definition $\Phi_{n}$ and $\Phi_{n}^{\delta}$ are invertible on the corresponding interval $\left[s_{i}^{n}, t_{i+1}^{n}\right]$. Hence denote $S_{n}(u)$ and $S_{n}^{\delta}(u)$ the reciprocal functions. 
To bound the next integral, we will make a change of variable from $s$ to $u=\Phi_{n}(s)$ and then go back to the original variable:

$$
\begin{aligned}
& \int_{\left[s_{i}^{n}, t_{i+1}^{n}\right]}\left(F^{0}\left(X(s)-\xi_{n} s\right)-F^{0}\left(X^{\delta}(s)-\xi_{n} s\right)\right) d s \\
& \leq \int_{\Phi_{n}\left(s_{i}^{n}\right)}^{\Phi_{n}\left(t_{i+1}^{n}\right)} F(u)\left(\frac{1}{\alpha\left(V\left(S_{n}(u)\right)\right)-\xi_{n} S_{n}(u)}-\frac{1}{\alpha\left(V^{\delta}\left(S_{n}^{\delta}(u)\right)\right)-\xi_{n} S_{n}^{\delta}(u)}\right) d u \\
& \quad+\|F\|_{\infty} \frac{\sup _{s}\left|X(s)-X^{\delta}(s)\right|}{a_{n} \eta} \\
& \leq C \frac{\|F\|_{\infty}}{a_{n} \eta} \int_{s_{i}^{n}}^{t_{i+1}^{n}}\left(\left|V(s)-V^{\delta}(s)\right|+\left|s-S_{n}^{\delta}\left(\Phi_{n}(s)\right)\right|\right) d s \\
& \quad+\|F\|_{\infty} \frac{\sup _{s}\left|X(s)-X^{\delta}(s)\right|}{a_{n} \eta}
\end{aligned}
$$

simply by using the Lipschitz regularity of $\alpha$ in $v$ and of $V, V^{\delta}$ in time. Next notice that

$$
\begin{aligned}
\left|s-S_{n}^{\delta}\left(\Phi_{n}(s)\right)\right| & =\mid S_{n}^{\delta}\left(\Phi_{n}^{\delta}(s)-S_{n}^{\delta}\left(\Phi_{n}(s)\right)\left|\leq\left\|S_{n}^{\delta}\right\|_{W^{1, \infty}}\right| \Phi_{n}^{\delta}(s)-\Phi_{n}(s) \mid\right. \\
& \leq C \frac{\sup _{s}\left|X(s)-X^{\delta}(s)\right|}{a_{n} \eta} .
\end{aligned}
$$

And hence deduce that

$$
\begin{aligned}
& \int_{\left[s_{i}^{n}, t_{i+1}^{n}\right]}\left(F^{0}\left(X(s)-\xi_{n} s\right)-F^{0}\left(X^{\delta}(s)-\xi_{n} s\right)\right) d s \leq C \frac{\|F\|_{\infty}}{a_{n} \eta} \\
& \left(\int_{s_{i}^{n}}^{t_{i+1}^{n}}\left|V(s)-V^{\delta}(s)\right| d s+\left(1+\left|t_{i+1}^{n}-s_{i}^{n}\right| /\left(a_{n} \eta\right)\right) \sup _{s}\left|X(s)-X^{\delta}(s)\right|\right) .
\end{aligned}
$$

Introducing this estimate, one obtains

$$
\begin{aligned}
\frac{d}{d t} R_{\delta}(T) \leq & C\|F\|_{\infty} \sum_{n} \mu_{n} \int_{0}^{t} \frac{\left|V(t)-V^{\delta}(t)\right|}{A_{\delta}(t, x, v)}\left(l_{n}+l_{n}^{\delta}\right. \\
+\frac{1}{a_{n} \eta} & \left.\int_{0}^{t}\left|V(s)-V^{\delta}(s)\right| d s+\frac{k_{n}+k_{n}^{\delta}+t /\left(a_{n} \eta\right)}{a_{n} \eta} \sup _{s \leq t}\left|X(s)-X^{\delta}(s)\right|\right) \\
& -2 \int_{0}^{T} \frac{\partial_{t} \bar{\delta}(t, x, v,|\delta|)}{A_{\delta}(t, x, v)} d t .
\end{aligned}
$$


Therefore

$$
\begin{aligned}
\frac{d}{d t} R_{\delta}(T) \leq & C\|F\|_{\infty} \sqrt{\log 1 /|\delta|} \sum_{n} \frac{\mu_{n}}{a_{n} \eta}\left(1+k_{n}+k_{n}^{\delta}+1 /\left(a_{n} \eta\right)\right) \\
& +\int_{0}^{T} \frac{\left|V(t)-V^{\delta}(t)\right| \sum_{n} \mu_{n}\left(l_{n}+l_{n}^{\delta}\right)-\partial_{t} \bar{\delta}}{A_{\delta}(t, x, v)} d t
\end{aligned}
$$

and from the definition (3.5) of $\bar{\delta}$ and the obvious bound $\left|V-V^{\delta}\right| \leq|\delta|+$ $T\|F\|_{\infty}$, one simply gets

$$
\frac{d}{d t} R_{\delta}(T) \leq C\|F\|_{\infty} \sqrt{\log 1 /|\delta|} \sum_{n} \frac{\mu_{n}}{a_{n} \eta}\left(1+k_{n}+k_{n}^{\delta}+1 /\left(a_{n} \eta\right)\right) .
$$

By the definition of the intervals and the fact that $V$ is Lipschitz in time

$$
\left|s_{i}^{n}-t_{i}^{n}\right| \geq a_{n} \eta / C \text {. }
$$

Hence

$$
l_{n} \geq k_{n} a_{n} \eta / C, \text { or } k_{n} \leq \frac{C T}{a_{n} \text { eta }} .
$$

So finally

$$
R_{\delta}(T) \leq C T\|F\|_{\infty} \frac{\sqrt{\log 1 /|\delta|}}{\eta^{2}} \sum_{n} \frac{\mu_{n}}{a_{n}^{2}} .
$$

Taking for instance $\eta=(\log 1 /|\delta|)^{-1 / 8}$, one indeed concludes the proof of Prop. 3.1, provided that it is possible to choose the $a_{n}$ s.t. $\sum_{n} \mu_{n} / a_{n}^{2}$ is finite.

The only other constraint to satisfy on the $a_{n}$ is that $\sum_{n} a_{n}<\infty$. By the bound (1.6), one may simply choose $a_{n}=n^{-\gamma / 2}$.

\section{References}

[1] G. Alberti, S. Bianchini, G. Crippa. A uniqueness result for the continuity equation in two dimensions, 2011. To appear Journal of the European Mathematical Society (JEMS).

[2] L. Ambrosio, Transport equation and Cauchy problem for $B V$ vector fields. Invent. Math. 158, 227-260 (2004). 
[3] D. Arsenio, L. Saint-Raymond. Personal communication, 2011.

[4] F. Bouchut, Renormalized solutions to the Vlasov equation with coefficients of bounded variation. Arch. Ration. Mech. Anal. 157 (2001), pp. $75-90$.

[5] F. Bouchut, L. Desvillettes, On two-dimensional Hamiltonian transport equations with continuous coefficients. Diff. Int. Eq. (8) 14 (2001), $1015-1024$.

[6] F. Bouchut, F. Golse, and C. Pallard. Classical solutions and the Glassey-Strauss theorem for the 3D Vlasov-Maxwell system. Arch. Ration. Mech. Anal., 170(1):1-15, 2003.

[7] N. Champagnat, P-E Jabin, Well posedness in any dimension for Hamiltonian flows with non $B V$ force terms. To appear Comm. Partial Differential Equations

[8] F. Colombini, G. Crippa, J. Rauch, A note on two-dimensional transport with bounded divergence. Comm. Partial Differential Equations 31 (2006), 1109-1115.

[9] F. Colombini, J. Rauch, Uniqueness in the Cauchy problem for transport in $\mathbb{R}^{2}$ and $\mathbb{R}^{1+2}$. J. Differential Equations 211 (2005), no. 1, 162167.

[10] G. Crippa, C. DeLellis, Estimates and regularity results for the DiPerna-Lions flow. J. Reine Angew. Math. 616 (2008), 15-46.

[11] C. De Lellis, Notes on hyperbolic systems of conservation laws and transport equations. Handbook of differential equations, Evolutionary equations, Vol. 3 (2007).

[12] N. De Pauw, Non unicité des solutions bornées pour un champ de vecteurs $B V$ en dehors d'un hyperplan. C.R. Math. Sci. Acad. Paris 337 (2003), 249-252.

[13] R.J. DiPerna, P.L. Lions, Ordinary differential equations, transport theory and Sobolev spaces. Invent. Math. 98 (1989), 511-547.

[14] R. J. DiPerna and P.-L. Lions. Global weak solutions of Vlasov-Maxwell systems. Comm. Pure Appl. Math., 42(6):729-757, 1989. 
[15] R. J. DiPerna and P.-L. Lions. On the Cauchy problem for Boltzmann equations: global existence and weak stability. Ann. of Math. (2), 130(2):321-366, 1989.

[16] P. Germain, N. Masmoudi. Global existence for the Euler-Maxwell system. Preprint, 2011.

[17] R. T. Glassey and W. A. Strauss. Singularity formation in a collisionless plasma could occur only at high velocities. Arch. Rational Mech. Anal., 92(1):59-90, 1986.

[18] M. Hauray, On two-dimensional Hamiltonian transport equations with $\mathbb{L}_{l o c}^{p}$ coefficients. Ann. IHP. Anal. Non Lin. (4) 20 (2003), 625-644.

[19] M. Hauray, On Liouville transport equation with force field in $B V_{\text {loc }}$. Comm. Partial Differential Equations 29 (2004), no. 1-2, 207-217.

[20] P.E. Jabin, Differential Equations with singular fields. J. de Math. Pures et Appl. (9) 94 (2010), no. 6, 597-621.

[21] S. Klainerman and G. Staffilani. A new approach to study the VlasovMaxwell system. Commun. Pure Appl. Anal., 1(1):103-125, 2002.

[22] C. Le Bris, P.L. Lions, Renormalized solutions of some transport equations with partially $W^{1,1}$ velocities and applications. Ann. Mat. Pura Appl. 183 (2004), 97-130.

[23] E.M. Stein, Maximal functions. I. Spherical means. Proc. Nat. Acad. Sci. U.S.A. 73 (1976), no. 7, 2174-2175. 